\title{
Determination of the anti-inflammatory and cytoprotective effects of L-glutamine and L-alanine, or dipeptide, supplementation in rats submitted to resistance exercise
}

\author{
Raquel Raizel ${ }^{1,2 *}$, Jaqueline Santos Moreira Leite ${ }^{1}$, Thaís Menezes Hypólito ${ }^{1}$, Audrey Yule Coqueiro ${ }^{1}$, \\ Philip Newsholme ${ }^{2}$, Vinicius Fernandes Cruzat ${ }^{2,3 *}$ and Julio Tirapegui ${ }^{1}$ \\ ${ }^{1}$ Department of Food Science and Experimental Nutrition, Faculty of Pharmaceutical Sciences, University of São Paulo, \\ São Paulo, SP 05508-000, Brazil \\ ${ }^{2}$ School of Biomedical Sciences, Faculty of Health Sciences and Curtin Health Innovation Research Institute, Curtin University, \\ WA 6102, Australia \\ ${ }^{3}$ Department of Physiology and Biophysics, Institute of Biomedical Sciences, University of São Paulo, São Paulo, SP 05508-000, \\ Brazil \\ (Submitted 21 December 2015 - Final revision received 9 April 2016 - Accepted 18 April 2016 - First published online 24 May 2016)
}

\section{Abstract}

We evaluated the effects of chronic oral supplementation with L-glutamine and L-alanine in their free form or as the dipeptide L-alanylL-glutamine (DIP) on muscle damage, inflammation and cytoprotection, in rats submitted to progressive resistance exercise (RE). Wistar rats ( $n$ 8/group) were submitted to 8-week RE, which consisted of climbing a ladder with progressive loads. In the final $21 \mathrm{~d}$ before euthanasia, supplements were delivered in a $4 \%$ solution in drinking water. Glutamine, creatine kinase (CK), lactate dehydrogenase (LDH), TNF- $\alpha$, specific IL (IL-1 $\beta$, IL-6 and IL-10) and monocyte chemoattractant protein-1 (MCP-1) levels were evaluated in plasma. The concentrations of glutamine, TNF- $\alpha$, IL- 6 and IL-10, as well as NF- $\kappa$ B activation, were determined in extensor digitorum longus (EDL) skeletal muscle. HSP70 level was assayed in EDL and peripheral blood mononuclear cells (PBMC). RE reduced glutamine concentration in plasma and EDL $(P<0 \cdot 05$ $v$. sedentary group). However, L-glutamine supplements (L-alanine plus L-glutamine (GLN + ALA) and DIP groups) restored glutamine levels in plasma (by 40 and 58\%, respectively) and muscle (by 93 and 105\%, respectively). GLN + ALA and DIP groups also exhibited increased level of HSP70 in EDL and PBMC, consistent with the reduction of NF- $\kappa$ B p 65 activation and cytokines in EDL. Muscle protection was also indicated by attenuation in plasma levels of CK, LDH, TNF- $\alpha$ and IL- $1 \beta$, as well as an increase in IL-6, IL-10 and MCP-1. Our study demonstrates that chronic oral L-glutamine treatment (given with L-alanine or as dipeptide) following progressive RE induces cyprotective effects mediated by HSP70associated responses to muscle damage and inflammation.

Key words: L-Alanyl-t-glutamine: Alanine: Resistance exercise: Inflammation: Heat-shock protein

The most abundant amino acid in the body, glutamine, is considered conditionally essential during stress and plays a key role in the inter-organ $\mathrm{N}$ transport ${ }^{(1)}$, intermediary metabolism $^{(2)}$, cellular redox pathways ${ }^{(1)}$, glucose ${ }^{(3)}$ and glutathione synthesis $^{(4)}$, as well as in several other essential metabolic processes $^{(5,6)}$. Glutamine is also an important modulator of the heat-shock protein (HSP) response, via O-glycosylation and phosphorylation of heat-shock factor 1 (HSF-1) ${ }^{(7,8)}$. HSP, especially the $70-\mathrm{kDa}$ family (HSP72 $+\mathrm{HSP} 73$ ), are proteins known as 'stress response proteins', as their expression is highly induced by different types of agents and catabolic stimuli, such as oxidative, thermal and metabolic stresses, infection and intense exercise ${ }^{(9)}$. Within the cell, HSP act as molecular chaperones maintaining cellular homoeostasis, protecting against injury and death ${ }^{(9)}$ and modulating inflammatory responses through the NF- $\kappa \mathrm{B}$ signalling pathway ${ }^{(10)}$. Recently, HSP70 was shown to act as a regulator of the early inflammatory response to muscle injury, because of its role in myofibre regeneration and recovery ${ }^{(11)}$.

In sport and exercise, glutamine supplementation is popular among athletes, as it is considered an important nutrient for immune function and muscle recovery from injury and

Abbreviations: ALA, trained supplemented with L-alanine; CK, creatine kinase; CTRL, trained control group; DIP, dipeptide L-alanyl-L-glutamine; EDL, extensor digitorum longus; GLN + ALA, L-alanine plus L-glutamine; HSP, heat-shock protein; LDH, lactate dehydrogenase; MCP-1, monocyte chemoattractant protein-1; PBMC, peripheral blood mononuclear cells; RE, resistance exercise; SED, sedentary group.

*Corresponding author: R. Raizel, email raqzel@usp.br; V. F. Cruzat, email vinifc@usp.br 
catabolism $^{(12)}$. In fact, glutamine is a critical fuel for immune cell function and proliferation ${ }^{(5,13)}$, and its major supply is from the skeletal muscle, which indicates a tight relationship between muscle and immune function. However, the efficacy of oral L-glutamine supplementation has raised many questions ${ }^{(4,13-17)}$. As studies in humans and animals have described the extensive intestinal metabolism of dietary glutamine ${ }^{(18,19)}$, the dipeptide L-alanyl-L-glutamine (DIP) has been utilised for several clinical $^{(20-22)}$ and sports nutrition studies ${ }^{(16,23-25)}$ as an alternative delivery form via oral administration. The effectiveness of DIP has been related to the intestinal peptide transporter 1 , which facilitates a wide broad of dipeptide and tripeptide absorption $^{(26,27)}$. The dipeptide also allows for a supply of more glutamine molecules at the physiological osmolality required for oral solutions ${ }^{(22)}$.

Studies in animal models subjected to intense and exhaustive aerobic exercise ${ }^{(4,28)}$, or subjected to infection ${ }^{(29,30)}$, have provided evidence that both DIP and free forms of L-glutamine, along with L-alanine supplementation, improve glutamine availability and mitigate muscle damage and related inflammation. Thus, L-alanine supplementation may be important for glutamine metabolism ${ }^{(24,31)}$. Considering that progressive resistance exercise (RE) is a commonly used form of exercise and the rat model can mimic human responses to exercise ${ }^{(32)}$, the purpose of the present study was to investigate the effects of chronic oral supplementation with L-glutamine and L-alanine, in their free or DIP forms, on muscle damage and inflammation in rats submitted to RE. We hypothesised that supplements containing L-alanine and L-glutamine could favour glutamine metabolism in skeletal muscle composed of fast-twitch fibres, such as the extensor digitorum longus (EDL), and increase the levels of HSP70 in both active muscle and circulating peripheral blood mononuclear cells (PBMC), attenuating the harmful inflammatory effects of heavy RE.

\section{Methods}

\section{Animals}

Male Wistar rats ( $n$ 40), aged 2 months and weighing 228.78 (sD 2.03 ) g, were obtained from the animal facility of the Faculty of Pharmaceutical Sciences at University of São Paulo and housed one per cage with free access to water and standard chow (NUVILAB CR1; Nuvital Nutrients), composed of $22 \%$ protein. The animals were kept under a $12 \mathrm{~h}$ light $-12 \mathrm{~h}$ dark cycle (lights on 16.00 hours, lights off 04.00 hours), at a room temperature of $22 \pm 2^{\circ} \mathrm{C}$ and relative humidity of $55 \pm 10 \%$, for 8 weeks. Food intake and body weight were registered three times per week, fluid intake was assessed daily and the final weight was determined before euthanasia.

All animals were allowed to adapt to the laboratory environment for 1 week before the beginning of the experimental protocol. After the adaptation period, the animals were weighed and randomly distributed into five groups ( $n$ 8/group): sedentary (SED), trained control (CTRL) and trained supplemented with L-alanine (ALA), L-alanine plus L-glutamine (GLN + ALA) or L-alanyl-L-glutamine (DIP). All procedures were approved by the Ethics Committee for Animal Experimentation of the Faculty of Pharmaceutical Sciences, University of São Paulo, according to the guidelines of the Brazilian College of Animal Experimentation (protocol number: CEUA/FCF/428).

\section{Resistance exercise protocol}

The 8-week exercise protocol was adapted from Scheffer et al. ${ }^{(33)}$, originally published by Hornberger \& Farrar ${ }^{(34)}$, which consisted of climbing a vertical ladder $(1 \cdot 1 \times 0 \cdot 18 \mathrm{~m}, 2 \mathrm{~cm}$ grid, $80^{\circ}$ inclined) with progressive loads secured to the base of the rats tail. During the adaptation period (2 weeks), all animals were familiarised to the apparatus carrying a load equal to $5 \%$ of the body weight. Then, the load training was progressively increased to $25,50,75$ and $100 \%$ of the body weight, involving three to six sets of eight repetitions, with 2-min break between sets. Each day of exercise was considered one session, and it was repeated every $48 \mathrm{~h}$.

Three tests of maximum carrying capacity (MCC) were performed to determine exercise performance as follows: after adaptation period (test 1), before supplementation (test 2) and after supplementation (test 3). Briefly, the test protocol consisted of a ladder climb with an initial load of $75 \%$ of body weight, plus an additional $30 \mathrm{~g}$ load on each climb until exhaustion, with $2 \mathrm{~min}$ of rest period. This procedure was successively repeated and the highest load carried was considered the MCC final result.

\section{Supplementation}

Supplements were diluted in the drinking water at a concentration of $4 \%$ ( $4 \mathrm{~g}$ dissolved in a final volume of $100 \mathrm{ml}$ ) and offered ad libitum in the last $21 \mathrm{~d}$ of the experiment. Administration in the drinking water was selected after technical difficulties in administering daily oral gavages and in an attempt to reduce the stress of manipulation, as well as to increase the frequency of amino acid intake throughout the day ${ }^{(35)}$. The total amount of amino acids in each administered supplement was calculated based on the commercial DIP concentration (Dipeptiven ${ }^{\circledR} ; 20 \mathrm{~g}$ of L-alanyl-L-glutamine dissolved in $100 \mathrm{ml}$ of water). Free L-glutamine and free L-alanine were manufactured by Labsynth (Synth), whereas Dipeptiven ${ }^{\circledR}$ was manufactured by Fresenius Kabi S.A. Supplement intake was measured daily.

\section{Plasma parameters and peripheral blood mononuclear cell isolation}

Blood lactate was determined during RE training every third session of each training load (25, 50, 75 and $100 \%$ of body weight). Samples were collected from the tail vein into heparinised tubes and assayed in a lactometer, Yellow Spring Instruments (YSI Life Science). A duration of $1 \mathrm{~h}$ after the last session of $\mathrm{RE}$, animals were killed by decapitation and blood was immediately collected into heparinized tubes and stored at $-80^{\circ} \mathrm{C}$.

In this study, we analysed the blood buffy coat composed of PBMC (lymphocytes, monocytes and dendritic cells). For PBMC isolation, fresh blood samples were diluted 1:1 in PBS/EDTA (2 $\mathrm{mm}$ ) and combined with an equal volume of Ficoll-Hypaque 
solution (Ficoll 400: F4375 and Hypaque: S4506; Sigma-Aldrich) according to the gradient separation method described in the study by Bøyum et al. ${ }^{(36)}$. Then, PBMC were aspirated and suspended on ice-cold radioimmunoprecipitation assay (RIPA) buffer containing protease and phosphatase inhibitors (Cell Signaling Technology).

Plasma activity of creatine kinase (CK) and lactate dehydrogenase (LDH) was determined with a commercial kit (Labtest). IL-1 $\beta$, IL-6, IL-10, TNF- $\alpha$ and monocyte chemoattractant protein-1 (MCP-1) levels were measured by the Luminex beads assay, using the Milliplex MAP Kit for Luminex 200 reader, according to the manufacturer's instructions (Millipore Corp.). Glutamine and glutamate concentrations were determined spectrophotometrically using a commercial kit (Sigma-Aldrich) adapted for microplate reader (Synergy H1 Hybrid wavelength; BioTek).

\section{Tissue measurements}

EDL skeletal muscle was surgically excised after euthanasia and immediately frozen in liquid $\mathrm{N}_{2}$ for subsequent analysis. Muscle glutamine and glutamate concentrations were determined as described by Lund ${ }^{(37)}$. Mean values were reported as micromoles of glutamine per gram of fresh tissue and as nmol of glutamine/mg of protein. Muscle protein content was quantified using the BCA Protein Assay kit (Pierce; Thermo Scientific). For IL-6, IL-10 and TNF- $\alpha$ analysis, muscle samples (250 mg) were homogenised in $1 \mathrm{ml}$ of PBS buffer containing protease and phosphatase inhibitors, and performed according to the immunoassay kit (Millipore).

Total DNA-binding activity of NF- $\kappa \mathrm{B}$ p 65 was determined in the nuclear extract of EDL muscle by electrophoretic mobility shift assay. Nuclear fractions were isolated and analysed as per the manufacturer's instructions using the NF- $\kappa \mathrm{B}$ p 50/p65 transcription factor assay kit (Abcam). NF- $\kappa \mathrm{B}$ p 65 was detected at $450 \mathrm{~nm}$. Data were shown as optical density normalised by corresponding nuclear protein concentration, which allows semi-quantitative comparisons of the relative amounts of nuclear NF- $\kappa \mathrm{B}$ p 65 among groups.

\section{Western blot analysis}

After homogenisation with RIPA buffer containing protease and phosphatase inhibitors, EDL skeletal muscle and PBMC lysates were combined with sample buffer containing $240 \mathrm{~mm}$-Tris (pH 6.8), $40 \%$ Glycerol, 0.8\% SDS, 200 mм- $\beta$-mercaptoethanol and $0.02 \%$ bromophenol blue. Amounts of protein $(40 \mu \mathrm{g})$ were subjected to SDS-PAGE and transferred onto nitrocellulose membranes (GE Healthcare).

Membranes were blocked with $0.5 \%$ bovine serum albumin (BSA; Sigma-Aldrich) diluted in PBS with Tween (PBST), washed and incubated at $4{ }^{\circ} \mathrm{C}$ with gentle shaking overnight, with primary antibody HSP70 1:1000 (Cell Signaling Technology). After three washes, membranes were incubated for $1 \mathrm{~h}$ with peroxidase-labelled anti-rabbit IgG antibodies (Cell Signaling Technology) diluted 1:5000 in PBST and 2\% BSA. Blots were visualised on ImageQuant 400 (GE Healthcare) with a 1:1 solution of ECL-Advance Western blotting Reagent (GE Healthcare). Monoclonal anti- $\beta$-actin-peroxidase antibody (Sigma-Aldrich) at a ratio of 1:25000 was used for gel loading control and protein normalisation. The densitometric analysis of the Western blot was performed by the protein level average, normalised to $\beta$-actin.

\section{Statistical analysis}

Comparisons among groups were carried out by one-way ANOVA with Tukey's honestly significant differences as a post hoc test. Analyses over multiple time points were performed with repeated measures. Pearson's correlation coefficient $(r)$ was used as a measure of association. The level of statistical significance was set at $P<0 \cdot 05$. Data were analysed using Prism 5.0 for windows and expressed as mean values and standard deviations.

\section{Results \\ Body weight, food and fluid intake}

Body weight gain and food intake were reduced (by 30 and $11 \%$, respectively) in trained CTRL compared with the SED group $(P<0.05)$. However, no differences were observed among trained groups before the supplementation period (data not shown). As depicted in Table 1, RE training attenuated body weight gain and food intake in CTRL group $(P<0.05 v$. SED) throughout the supplementation period, and no difference was found in water intake between SED and CTRL groups. Although supplement intake was higher in ALA $(65 \cdot 14(\mathrm{SD} 2 \cdot 92) \mathrm{ml} / \mathrm{d})$ than in GLN + ALA and DIP groups (60.15 (SD 1.90) and $52 \cdot 75$

Table 1. Body weight gain, food and fluid intake of rats submitted to resistance exercise and evaluated during 3-week supplementation period§ (Mean values and standard deviations; $n$ 8)

\begin{tabular}{|c|c|c|c|c|c|c|c|c|c|c|}
\hline & \multicolumn{2}{|c|}{ SED } & \multicolumn{2}{|c|}{ CTRL } & \multicolumn{2}{|c|}{ ALA } & \multicolumn{2}{|c|}{$\mathrm{GLN}+\mathrm{ALA}$} & \multicolumn{2}{|c|}{ DIP } \\
\hline & Mean & SD & Mean & SD & Mean & SD & Mean & SD & Mean & SD \\
\hline Body weight gain (\%) & $13 \cdot 11$ & 1.77 & $7 \cdot 17^{\star}$ & $2 \cdot 17$ & $6 \cdot 28^{*}$ & 3.22 & $7.35^{\star}$ & 2.80 & $8 \cdot 11^{*}$ & 1.72 \\
\hline Food intake $(\mathrm{g} / \mathrm{d})$ & 27.08 & 0.65 & $23.00^{*}$ & 1.92 & $22 \cdot 15^{\star}$ & 2.04 & $21.69^{*}$ & 0.53 & $21.66^{*}$ & 0.32 \\
\hline Fluid intake $(\mathrm{ml} / \mathrm{d})$ & 39.06 & $2 \cdot 12$ & 37.88 & $2 \cdot 16$ & $65 \cdot 14^{\star \dagger \ddagger}$ & $7 \cdot 16$ & $60 \cdot 15^{\star \dagger}$ & 4.64 & $52 \cdot 75^{\star \dagger}$ & 4.66 \\
\hline
\end{tabular}

SED, sedentary group; CTRL, trained control; ALA, trained supplemented with L-alanine; GLN + ALA, trained supplemented with L-glutamine plus L-alanine in free form; DIP, trained supplemented with L-alanyl-L-glutamine.

* $P<0.05$ v. SED.

$\dagger P<0.05$ v. CTRL.

$\ddagger P<0.05$ v. DIP.

$\S$ SED and CTRL received water and supplements given in a $4 \%$ solution. 


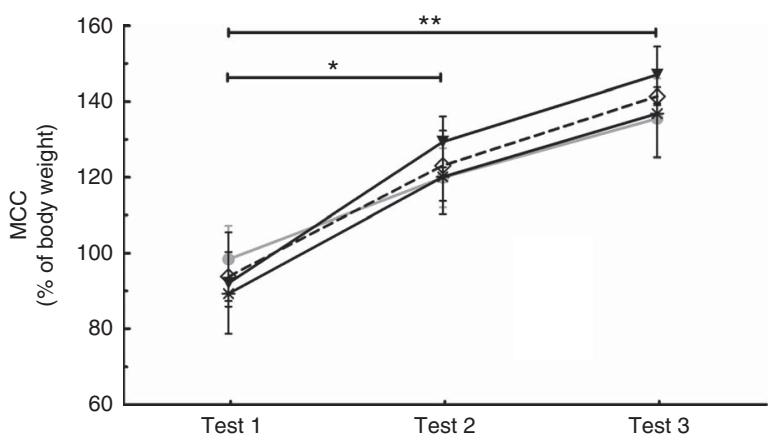

Fig. 1. Maximum carrying capacity (MCC) test was performed before supplementation (test 1 and test 2) and after supplementation period (test 3 ) over the course of resistance exercise protocol. Values are means $(n 8)$, and standard deviations. ${ }^{*} P<0.05$ test $1 \mathrm{~V}$. test 2 ; ${ }^{\star *} P<0.01$ test $1 \mathrm{v}$. test 3 . - - Trained control group; $\rightarrow$, trained supplemented with L-alanine; $\diamond$, L-alanine plus L-glutamine; $\longrightarrow$, dipeptide L-alanyl-L-glutamine.

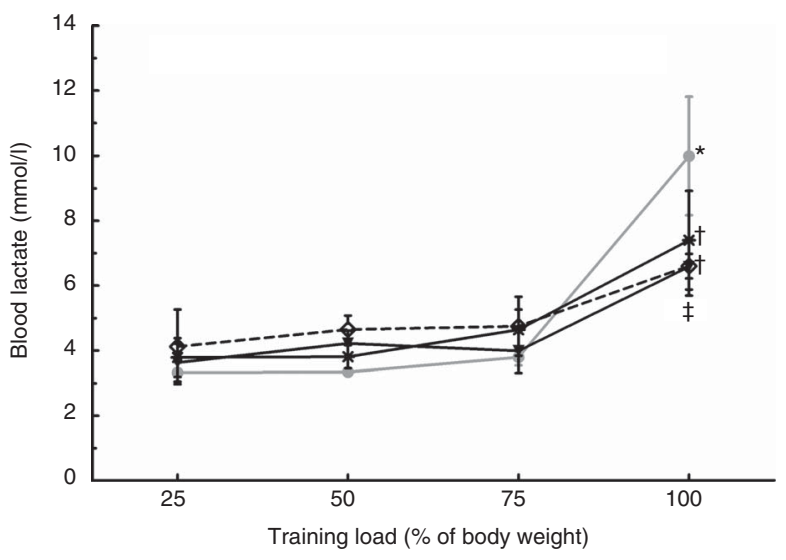

Fig. 2. Levels of blood lactate in rats submitted to progressive resistance training. Lactate was assessed in four time points, every third session of each training load (25, 50, 75 and $100 \%$ of body weight). Trained control group

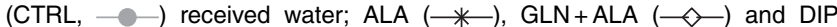
$(-\boldsymbol{\nabla})$ were supplemented with L-alanine, L-alanine plus L-glutamine and L-alanyl-L-glutamine, respectively, in a $4 \%$ solution. Values are means $(n 8)$, and standard deviations. ${ }^{*} P<0.05 \mathrm{v} .25,50$ and $75 \%$ of BW load; ${ }^{\dagger} P<0.05 \mathrm{v}$. $25 \%$ of BW load; ${ }^{\ddagger} P<0.05$ GLN + ALA and DIP $v$. CTRL. ALA, Trained supplemented with L-alanine; GLN + ALA, L-glutamine plus L-alanine; DIP, dipeptide L-alanyl-L-glutamine.

(SD 1.90$) \mathrm{ml} / \mathrm{d}$, respectively), as well as in all supplemented groups compared with CTRL $(P<0.05)$, L-glutamine or L-alanine administrations did not affect weight gain and food intake.

\section{Maximum carrying capacity performance test and the lactate response}

The MCC test represents a method to determine exercise performance $^{(34)}$. As depicted in Fig. 1, all trained animals demonstrated increased performance $(P<0.05)$ in tests 2 and 3 (approximately 32 and $50 \%$, respectively) when compared with the first test. However, it is noteworthy that no difference was observed between test 2 and test 3, which suggests a lower carrying capacity induced by high-intensity RE. Although L-glutamine is expected to be useful to replenish amino acid body stores, it is unlikely that this nutritional supplementation can enhance performance, as previously reported ${ }^{(15,38,39)}$. In fact, the MCC test results demonstrated no significant difference between controls and supplemented animals (Fig. 1).

Lactate concentration was determined in four different time points to evaluate exercise intensity and metabolic demand. As illustrated in Fig. 2, controls exhibited blood lactate 2-fold higher at a load of $100 \%$ compared with all previous loads $(P<0.05)$, suggesting increased energy demand promoted by $\mathrm{RE}$. In the supplemented groups, lactate at $100 \%$ of BW increased approximately $80 \%$, which was statistically significant when compared with the $25 \%$ load $(P<0.05)$. Moreover, both L-glutamine-supplemented groups were associated with lower lactate concentration at $100 \%$, when compared with the controls $(P<0 \cdot 05)$.

\section{Glutamine metabolism in plasma and skeletal muscle}

Because of the increased requirements triggered by heavy exercise training, the concentration of glutamine in plasma and tissues falls sharply, especially in major stores of the amino acid for the whole body, such as the skeletal muscle. The results presented herein demonstrated that RE training compromised both plasma (Table 2) and muscle (Table 3) glutamine levels, and the muscle glutamine:glutamate ratio (Table 3 ) in the controls, when compared with SED animals $(P<0.05)$. In contrast, the nutritional interventions with ALA, GLN + ALA and DIP reduced the severity of RE effects, increasing plasma glutamine concentration (by 45 , 40 and $57 \%$, respectively), when compared with the CTRL group $(P<0.05)$. Moreover, muscle glutamine concentrations were significantly restored (both $\mu \mathrm{mol} / \mathrm{g}$ fresh tissue and $\mathrm{nmol} / \mathrm{mg}$ protein) in GLN + ALA and DIP groups (Table 3).

Although the amino acid intake was increased in supplemented groups (by 33\%) compared with CTRL, no significant correlation was observed between amino acid intake and the concentrations of glutamine in plasma of ALA $(r-0 \cdot 28$, $P=0.59)$, GLN + ALA $(r-0.42, P=0.40)$ and DIP $(r-0.40$, $P=0.42)$ groups, as well as in muscle $(r$ 0.04, $P=0.93$; $r 0 \cdot 33, P=0 \cdot 52 ; r 0 \cdot 10, P=0 \cdot 85$, respectively).

\section{Muscle damage and inflammation in plasma and skeletal muscle}

Assessing plasma markers of muscle damage involved determination of the activity of CK and LDH. This was high in the controls (56 and 39\%, respectively) as compared with the SED group (Table $2, P<0.05$ ). However, this effect was attenuated by GLN + ALA and DIP supplementation, when compared with the CTRL group $(P<0.05)$.

Besides elevations in CK and LDH, RE increased plasma IL- $1 \beta$ by $75 \%$, TNF- $\alpha$ by $14 \%$, IL- 6 by $45 \%$, IL- 10 by $46 \%$ and MCP- 1 by $33 \%$ in the CTRL group as compared with SED $(P<0.05)$. Nevertheless, nutritional interventions with ALA, GLN + ALA and DIP reduced plasma concentrations of IL- $1 \beta$ and TNF- $\alpha$ (by 57 and $21 \%$, respectively), when compared with controls $(P<0.05)$. Interestingly, animals supplemented with GLN + ALA and DIP exhibited an increase in the concentration of IL-6 
Table 2. Glutamine, glutamate and markers of muscle damage and inflammation in plasma of rats submitted to 8-week resistance exercise§ (Mean values and standard deviations; $n$ 8)

\begin{tabular}{|c|c|c|c|c|c|c|c|c|c|c|}
\hline & \multicolumn{2}{|c|}{ SED } & \multicolumn{2}{|c|}{ CTRL } & \multicolumn{2}{|c|}{ ALA } & \multicolumn{2}{|c|}{$\mathrm{GLN}+\mathrm{ALA}$} & \multicolumn{2}{|c|}{ DIP } \\
\hline & Mean & SD & Mean & SD & Mean & SD & Mean & SD & Mean & SD \\
\hline $\mathrm{G} \ln (\mu \mathrm{mol} / \mathrm{l})$ & 1.15 & 0.03 & $0.99^{*}$ & $0 \cdot 10$ & $1.44^{\dagger}$ & 0.11 & $1.39^{\dagger}$ & 0.20 & $1.56^{\star \dagger}$ & 0.37 \\
\hline Glu $(\mu \mathrm{mol} / /)$ & 0.83 & 0.25 & 0.79 & 0.31 & 0.76 & 0.27 & 0.79 & 0.30 & 0.73 & 0.36 \\
\hline $\mathrm{CK}(\mathrm{U} / \mathrm{ml})$ & $12 \cdot 10$ & 2.23 & $18 \cdot 83^{*}$ & 2.62 & $16 \cdot 32^{\ddagger}$ & $5 \cdot 20$ & $10 \cdot 27^{\dagger}$ & 4.60 & $9 \cdot 82^{\dagger}$ & 3.03 \\
\hline LDH (U/ml) & $5 \cdot 13$ & 0.77 & $7 \cdot 12^{*}$ & 1.33 & $5 \cdot 30$ & 1.65 & $4.55^{\dagger}$ & 1.35 & $4.77^{\dagger}$ & 1.08 \\
\hline $\mathrm{IL}-1 \beta(\mathrm{pg} / \mathrm{ml})$ & 0.08 & 0.03 & $0.14^{\star}$ & 0.03 & $0.07^{\dagger}$ & 0.04 & $0.06^{\dagger}$ & 0.04 & $0.05^{\dagger}$ & 0.03 \\
\hline TNF-a (pg/ml) & 1.77 & 0.02 & $2.05^{\star}$ & 0.07 & $1.68^{\dagger}$ & 0.09 & $1.69^{\dagger}$ & 0.08 & $1.52^{\dagger}$ & 0.08 \\
\hline IL-6 (pg/ml) & 7.38 & $2 \cdot 10$ & $10 \cdot 72^{*}$ & $2 \cdot 30$ & $11 \cdot 18^{\star \neq}$ & 1.51 & $14.92^{\star \dagger}$ & 2.65 & $14 \cdot 70^{\star \dagger}$ & 2.08 \\
\hline IL-10 (pg/ml) & 0.95 & 0.23 & $1.39^{\star}$ & 0.14 & $1.53^{\star \ddagger}$ & 0.52 & $2 \cdot 67^{\star \dagger}$ & 0.48 & $2 \cdot 64^{\star \dagger}$ & 0.35 \\
\hline MCP-1 (pg/ml) & 309.72 & $85 \cdot 86$ & $412 \cdot 9^{\star}$ & 64.87 & $432 \cdot 80^{\star}$ & 37.52 & $518 \cdot 10^{\star \dagger}$ & 69.95 & $533.90^{\star} \dagger$ & 88.81 \\
\hline
\end{tabular}

SED, sedentary group; CTRL, trained control; ALA, trained supplemented with L-alanine; GLN + ALA, trained supplemented with L-glutamine plus L-alanine in free form; DIP, trained supplemented with L-alanyl-L-glutamine; CK, creatine kinase; LDH, lactate dehydrogenase; MCP-1, monocyte chemoattractant protein-1.

* $P<0.05$ v. SED.

$\dagger P<0.05$ v. CTRL

$\ddagger P<0.05$ v. GLN + ALA and DIP.

$\S$ SED and CTRL received water and supplements given in a $4 \%$ solution.

Table 3. Skeletal muscle contents of glutamine and glutamate and inflammation markers of rats submitted to 8-week resistance exercise (Mean values and standard deviations; $n$ 8)

\begin{tabular}{|c|c|c|c|c|c|c|c|c|c|c|}
\hline & \multicolumn{2}{|c|}{ SED } & \multicolumn{2}{|c|}{ CTRL } & \multicolumn{2}{|c|}{ ALA } & \multicolumn{2}{|c|}{$G L N+A L A$} & \multicolumn{2}{|c|}{ DIP } \\
\hline & Mean & SD & Mean & SD & Mean & SD & Mean & SD & Mean & SD \\
\hline Gln ( $\mu \mathrm{mol} / \mathrm{g}$ fresh tissue) & 8.59 & 0.21 & $6 \cdot 73^{*}$ & 0.75 & 8.00 & 1.43 & $8 \cdot 77^{\dagger}$ & 0.52 & $9.05^{\dagger}$ & 1.05 \\
\hline Glu ( $\mu \mathrm{mol} / \mathrm{g}$ fresh tissue) & $6 \cdot 18$ & 0.45 & $6 \cdot 26$ & $0 \cdot 10$ & $6 \cdot 28$ & $0 \cdot 10$ & $6 \cdot 44$ & 0.25 & $6 \cdot 32$ & 0.08 \\
\hline GIn/Glu & 1.40 & 0.06 & $0.99^{*}$ & 0.21 & $1 \cdot 27$ & 0.26 & $1 \cdot 31^{\dagger}$ & 0.15 & $1.37^{\dagger}$ & 0.24 \\
\hline Gln (nmol/mg protein) & $20 \cdot 46$ & 3.27 & $11.94^{*}$ & 4.23 & 22.44 & $11 \cdot 12$ & $23.06^{\dagger}$ & 2.55 & $24.51^{\dagger}$ & $10 \cdot 63$ \\
\hline Glu (nmol/mg protein) & 13.98 & 4.92 & $18 \cdot 23$ & 8.08 & $18 \cdot 80$ & $7 \cdot 41$ & $17 \cdot 94$ & $2 \cdot 48$ & 14.45 & 3.66 \\
\hline Gln/Glu & 1.41 & 0.04 & $0.99^{\star}$ & 0.05 & $1 \cdot 27$ & 0.34 & $1 \cdot 31^{\dagger}$ & 0.17 & $1.33^{\dagger}$ & 0.23 \\
\hline TNF- $a$ (pg/mg protein) & 0.12 & 0.01 & $0.14^{*}$ & 0.01 & $0.10^{\dagger}$ & 0.02 & $0.09^{\dagger}$ & 0.02 & $0.09^{\dagger}$ & 0.02 \\
\hline IL-6 (pg/mg protein) & $6 \cdot 61$ & 0.75 & $8 \cdot 35^{\star}$ & 0.98 & $6 \cdot 11^{\dagger}$ & $1 \cdot 17$ & $6 \cdot 11^{\dagger}$ & 1.07 & $5.93^{\dagger}$ & $1 \cdot 10$ \\
\hline IL-10 (pg/mg protein) & 0.60 & 0.18 & $1 \cdot 13^{*}$ & 0.20 & $0.62^{\dagger}$ & 0.24 & $0.61^{\dagger}$ & 0.19 & $0.58^{\dagger}$ & 0.07 \\
\hline
\end{tabular}

SED, sedentary group; CTRL, trained control; ALA, trained supplemented with L-alanine; GLN + ALA, trained supplemented with L-glutamine plus L-alanine in free form; DIP, trained supplemented with L-alanyl-L-glutamine.

${ }^{*} P<0.05$ v. SED.

$\dagger P<0.05$ v. CTRL.

¥ SED and CTRL received water and supplements given in a $4 \%$ solution.

(38\%), IL-10 (91\%) and MCP-1 (28\%), as compared with the CTRL group (Table 2, $P<0 \cdot 05$ ).

As depicted in Table 3, both RE training and nutritional supplementation had an impact on muscle inflammation. In the CTRL group, inflammatory mediators such as TNF- $\alpha$, IL- 6 and IL-10 increased because of the RE training protocol, when compared with SED animals $(P<0.05)$. However, ALA, GLN + ALA and DIP supplements attenuated this finding $(P<0.05 v$. CTRL group), maintaining the concentration of muscle TNF- $\alpha$, IL-6 and IL-10 close to basal levels observed in the SED group.

\section{Glutamine effects on HSP70 response and muscle $N F-\kappa B$ activation}

HSP, especially the HSP70 family (the major HSP), provide critical protection against several forms of injury. Although exercise is a potent stimulus for the HSP response, local and systemic inflammatory injury leads to a deficit in HSP70 protein levels, which may impair recovery. Herein, muscle HSP70 level was markedly reduced (by $51 \%$ ) by RE training in the controls, as compared with SED animals (Fig. 3, $P<0.05$ ). Moreover, RE produced a severe decrease (by 88\%) in HSP70 levels in circulating PBMC (Fig. 4, $P<0.05$ ) possibly because these cells are unable to synthesise glutamine and are largely dependent on plasma and muscle glutamine availability, which is compromised by RE. In fact, glutamine availability is critical for the optimal regulation of HSP response. Indeed, GLN + ALA and DIP supplements were able to restore HSP70 concentration in both EDL skeletal muscle (Fig. 3) and PBMC (Fig. 4), when compared with CTRL animals $(P<0 \cdot 05)$.

Intracellular HSP70 have important anti-inflammatory properties, providing stress tolerance by blocking the activation of the NF- $\kappa \mathrm{B}$ pathway ${ }^{(30,40)}$. Our results with RE training are in agreement with in vitro and aerobic exercise studies, since decreased HSP70 levels promoted inflammatory effects by enhancing the activation of NF- $\kappa$ B p 65 in the skeletal muscle of controls by $34 \%$, as compared with the SED group (Fig. 5, $P<0.05$ ). Moreover, glutamine is a key substrate that has an impact on HSP70 expression. Consequently, the increased HSP70 levels found in EDL reduced (by $30 \%$ ) NF- $\kappa \mathrm{B}$ p65 activation, when compared with controls $(P<0 \cdot 05)$. 

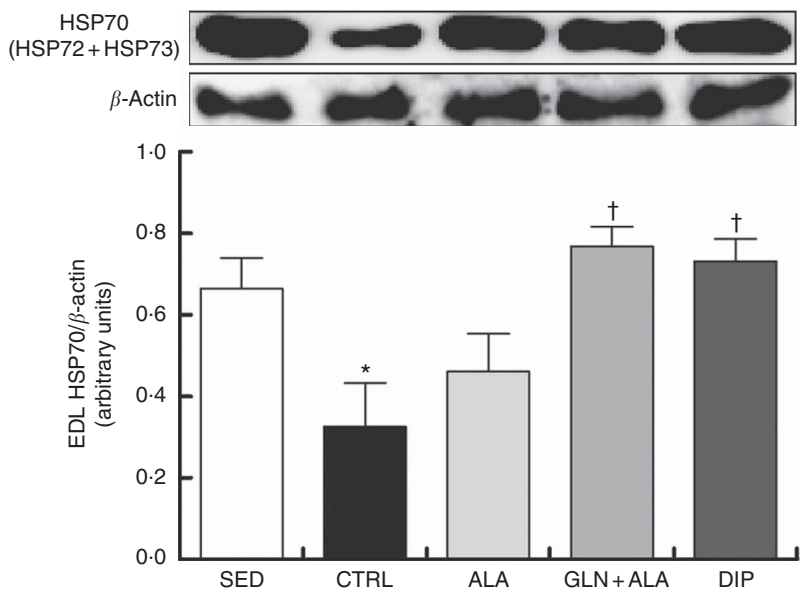

Fig. 3. HSP70 protein levels in extensor digitorum longus (EDL) muscle of rats submitted to resistance exercise. Sedentary (SED) and trained control (CTRL) groups received water; ALA, GLN + ALA and DIP groups were submitted to 8-week resistance exercise and supplemented with L-alanine, L-alanine plus L-glutamine and L-alanyl-L-glutamine, respectively, in a $4 \%$ solution. Supplements were given ad libitum in the last $21 \mathrm{~d}$ of the experiment. EDL muscle was excised and immediately frozen in liquid $\mathrm{N}_{2}$ for further analysis. Homogenates were immunoblotted for HSP70 and bands were normalised with $\beta$-actin controls. Values are means $(n 8)$, and standard deviations represented by vertical bars. ${ }^{*} P<0.05 \mathrm{v}$. SED; ${ }^{\dagger} P<0.05 \mathrm{v}$. CTRL. ALA, Trained supplemented with L-alanine; GLN + ALA, L-glutamine plus L-alanine; DIP, dipeptide L-alanyl-L-glutamine.

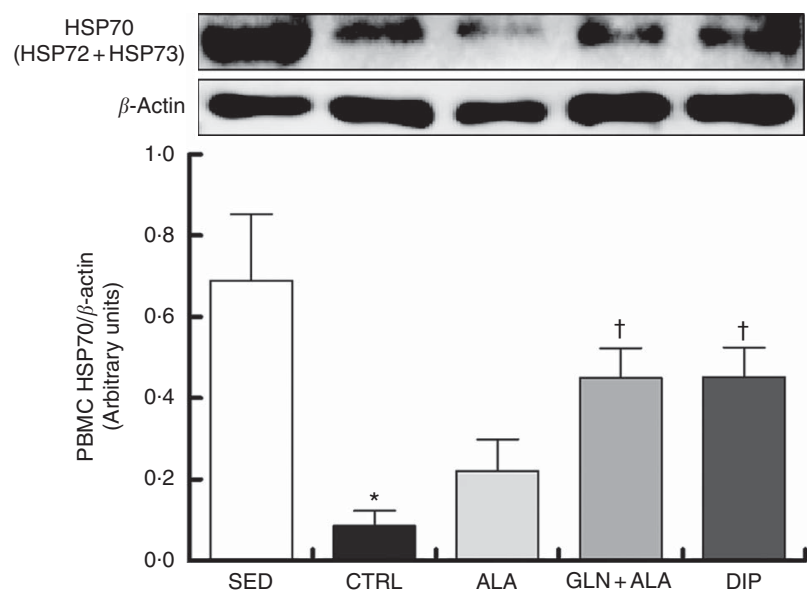

Fig. 4. HSP70 protein levels in peripheral blood mononuclear cells (PBMC) of rats submitted to resistance exercise. Sedentary (SED) and trained control (CTRL) groups received water; ALA, GLN + ALA and DIP groups were submitted to 8-week resistance exercise and supplemented with L-alanine, L-alanine plus L-glutamine and L-alanyl-L-glutamine, respectively, in a $4 \%$ solution. Supplements were given ad libitum in the last $21 \mathrm{~d}$ of the experiment. Fresh blood samples were collected and peripheral blood mononuclear cells (PBMC) were separated by Ficoll density gradient centrifugation. Homogenates were immunoblotted for HSP70 and bands normalised with $\beta$-actin controls. Values are means $(n 8)$, and standard deviations represented by vertical bars. * $P<0.05$ v. SED; ${ }^{\dagger} P<0.05$ v. CTRL. ALA, Trained supplemented with L-alanine; GLN + ALA, L-glutamine plus L-alanine; DIP, dipeptide L-alanyl---glutamine.

\section{Discussion}

Our study demonstrated that chronic oral L-glutamine treatment (in free form along with $\mathrm{L}$-alanine or as dipeptide) following progressive RE promotes cyprotective effects involving HSP70

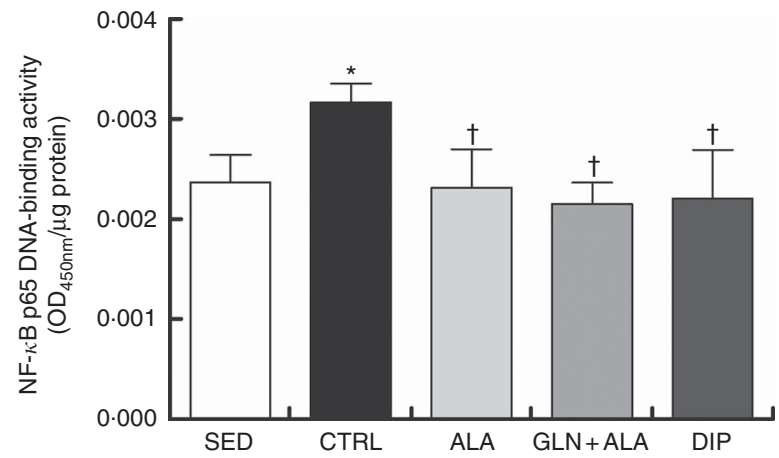

Fig. 5. NF- $\kappa$ B p65 DNA-binding activity in the nuclear extract of extensor digitorum longus muscle of rats submitted to resistance exercise. Sedentary (SED) and trained control (CTRL) groups received water; ALA, GLN + ALA and DIP groups were submitted to 8-week resistance exercise and supplemented with L-alanine, L-alanine plus L-glutamine and L-alanyl-L-glutamine, respectively, in a $4 \%$ solution. Supplements were given ad libitum in the last $21 \mathrm{~d}$ of the experiment. EDL muscle was excised and frozen in liquid $\mathrm{N}_{2}$ for further preparation of nuclear extract. Values are means $(n 8)$, and standard deviations represented by vertical bars. ${ }^{*} P<0.05$ v. SED; ${ }^{\dagger} P<0.05$ v. CTRL. ALA, Trained supplemented with L-alanine; GLN + ALA, L-glutamine plus L-alanine; DIP, dipeptide L-alanyl-L-glutamine.

responses, thus reducing muscle damage and inflammation. Glutamine is widely accepted as an important amino acid for cell metabolism and function, serving as a key metabolic substrate to provide a fuel source and aid $\mathrm{N}$ balance ${ }^{(18,19)}$. Glutamine metabolism and blood concentration is dramatically compromised under catabolic situations, which include high intensity and long periods of exercise. Surprisingly, little data exist on glutamine metabolism following exogenous supply in predominantly anaerobic activities, such as RE training.

As described herein, RE resulted in lower glutamine concentrations in plasma and EDL muscle. Muscle tissue is quantitatively the largest biosynthetic source and is essential for glutamine production and release, influencing plasma glutamine concentration, as well as its utilisation by other tissues and cells such as $\mathrm{PBMC}^{(5,13,24,41)}$. Although glutamine accounts for at least $50 \%$ of the free pool of amino acids in skeletal muscle, under catabolic situations the release of glutamine from muscle exceeds synthesis, and it may impair recovery from muscle damage because of the enhanced rate of protein breakdown induced by $\mathrm{N}$ imbalance following exercise ${ }^{(1,5,24)}$. In the current study, chronic oral administration with L-glutamine and L-alanine, in their free form or as the dipeptide, did not influence food intake and body weight gain, which allowed us to evaluate the exclusive effect of supplements in trained rats. The beneficial effects described here are supportive of results published in other studies ${ }^{(4,12,16,23,29)}$.

Interestingly, L-alanine co-supplementation significantly increased glutamine concentration and effects. Exercise is characterised by a shift in blood flow from the gastrointestinal tract to the active muscle, which may lead to changes in intestinal absorption of glutamine, as demonstrated in catabolic conditions ${ }^{(19)}$. The presence of alanine in supplements containing glutamine has been shown to alter glutamine metabolism, as it is metabolised via alanine aminotransferase to pyruvate, and rapidly consumed in the Krebs Cycle to generate ATP and glutamate production from 2 -oxoglutarate $e^{(23,42,43)}$. Similar to 
glutamine, alanine has a central role for maintenance of intermediary metabolism ${ }^{(24)}$. Elevated alanine release from muscle observed during exercise and following training has been considered necessary to provide a non-toxic form of $\mathrm{N}$ transport from muscle and a substrate for gluconeogenesis in the liver, as glycolytic flux is increased proportionally to the work intensity during muscle contractions ${ }^{(44)}$. Thus, despite the fact that high energy demand evidenced by blood lactate increases in this study, the high glutamine concentration in plasma of rats treated with L-alanine supports the concept that alanine supplementation may supply extra substrate for liver and kidney gluconeogenesis sparing glutamine. Oral supplementation with glutamine given in combination with other amino acids has beneficial effects on reducing inflammatory cytokine levels ${ }^{(19)}$. Herein, we have described the beneficial effects of L-alanine supplementation by reducing pro-inflammatory cytokines; however, administration of L-alanine alone did not enhance the glutamine content of skeletal muscle of trained rats.

Glutamine has also been used as an enhancer of HSP $70 \mathrm{kDa}$ (HSP70) levels ${ }^{(23,45)}$ via O-glycosylation and phosphorylation of HSF- $1^{(7,8)}$ in both stressed and unstressed animals ${ }^{(45)}$. HSP of $70 \mathrm{kDa}$ (HSP70) is one of the most inducible HSP isoforms and interacts with other proteins in an ATP-dependent manner, induced by various stimuli such as exercise, muscle injury and regeneration $^{(9,46)}$. HSP70 has been considered to play an important role in regulating skeletal muscle plasticity ${ }^{(23,46,47)}$, apoptosis and cell death by affecting protein folding, ubiquitin degradation pathways and protein translocation ${ }^{(48)}$. Recently, a chaperone balance hypothesis has been proposed. The activation of NF- $\kappa$ B can result in lower levels of intracellular HSP70, releasing pro-inflammatory extracellular HSP70, which may act to reduce oxidative stress in target cells. However, when chronically elevated, extracellular HSP70 stimulates inflammation, oxidative stress, reduction of HSF-1 expression and eventually reduced intracellular $\mathrm{HSP} 70^{(49)}$.

In order to investigate the heat-shock response following progressive RE training, a common form of exercise, we evaluated HSP70 protein levels in fast-twitch EDL muscle, a type of muscle that can generate high-power outputs during exercise ${ }^{(50)}$. Although exercise is known to stimulate the HSP response, we report herein that total HSP70 content in EDL muscle markedly decreased in the CTRL group, which may have been a consequence of impairment of glutamine availability and activation of NF- $\kappa$ B. The suppression of HSP response was also found in PBMC, a critical component of the immune system involved in the muscle repair process after exercise, as these cells are a primary source of pro-inflammatory cytokine release ${ }^{(51-53)}$. PBMC are largely dependent on skeletal muscle glutamine synthesis and release into blood, as these cells do not possess glutamine synthetase to catalyse glutamine synthesis from ammonia and glutamate $^{(15)}$. Glutamine may directly modulate pro-inflammatory cytokine release in PBMC, which may be related to the heatshock response ${ }^{(53)}$. Herein, treatments with DIP and GLN + ALA evoked a cytoprotective response to damaged tissue mediated by replenishment of glutamine concentrations, besides increased HSP70 levels in skeletal muscle and PBMC.

HSP70 has been considered a regulator of the early inflammatory response to muscle injury because of its beneficial role in myofibre regeneration and recovery ${ }^{(11)}$. The increase of HSP70 results in the inhibition of inflammatory cytokine production in human $\mathrm{PBMC}^{(53)}$, as well as by inactivation of the $\mathrm{NF}-\kappa \mathrm{B}$ signalling pathway ${ }^{(10,46,54)}$. Our results suggest that HSP70 high levels attenuated the production of inflammatory cytokines by inactivation of NF- $\kappa \mathrm{B}$. Inflammatory responses are mediated by activation of critical signalling pathways, such as $\mathrm{NF}-\kappa \mathrm{B}^{(10,55-57)}$. In a single acute exercise bout, the NF- $\kappa \mathrm{B}$ activity in skeletal muscle of rats is increased ${ }^{(58,59)}$. The $\mathrm{NF}-\kappa \mathrm{B}$ pathway acts as a central integrator of responses to mechanical, oxidative and inflammatory stress ${ }^{(54)}$. However, continuous activation and production of inflammatory components can stimulate excessive recruitment of immune cells, promoting further tissue injury ${ }^{(60)}$. Our study demonstrated an increase in NF- $\kappa$ B p 65 nuclear activity in EDL muscle of CTRL animals. Conversely, all nutritional treatments attenuated the effect of RE. Glutamine can attenuate the activation of multiple pathways of inflammation such as $\mathrm{NF}-\kappa \mathrm{B}$ through the ubiquitin-proteasome pathway, conferring protection against exacerbated inflammatory conditions ${ }^{(57,61)}$.

A decrease in glutamine levels is well established in stressful situations, such as high intensity and prolonged exercise (resulting in severe damage), as well as activation of inflammatory processes, raising the rate of protein degradation $^{(3,4,12,13,16,23)}$. In this study, RE induced an increase in plasma levels of CK and LDH enzymes. Despite the fact that CK and $\mathrm{LDH}$ are known as late markers of cellular injury, our exercise protocol was able to promote significant changes in $1 \mathrm{~h}$ after the last exercise session. A recent study ${ }^{(62)}$ has demonstrated that eccentric contractions induce a significant increase in muscle CK efflux immediately after exercise. Mechanical contractions produced by RE can induce micro-traumas in muscle fibres promoting breakdown of the extracellular matrix, basal lamina and sarcolemma, which leads to changes in membrane structure and permeability ${ }^{(55)}$. CK is located almost exclusively in brain, skeletal and cardiac muscle and is released to the bloodstream after damage to the structure of non-contractile muscle elements, which is induced by intense exercise ${ }^{(56)}$. Moderate bouts of eccentric exercise can induce physiological muscle adaptation; however, repeated intense exercise may induce higher release of muscle damage markers and loss of muscle proteins ${ }^{(63-66)}$. This was observed in the CTRL group, which indicates additional muscle damage after each exercise session.

Despite harmful effects of RE training, there was a decline in plasma CK and LDH activity in groups supplemented with glutamine (GLN + ALA; DIP), suggesting that muscle became more resistant to subsequent injury caused by a new session of RE. A recent study showed inhibition of signalling proteins that activate protein degradation after acute RE in rats supplemented with L-alanylglutamine ${ }^{(67)}$, and previous studies published by our group indicated that L-glutamine-containing supplements represent an effective way to maintain L-glutamine levels, which results in attenuation of the release of substances indicative of muscle damage and oxidative stress in trained rats ${ }^{(4,12,23)}$

The increase of lactate induced by high metabolic demand in RE promotes mobilisation of WBC into the circulation ${ }^{(51)}$. Muscle micro-injury also induces an influx of macrophages from the circulation in order to promote muscle repair and remodelling, through production of pro- and anti-inflammatory 
cytokines $^{(52,68,69)}$. However, continuous injuries without adequate rest periods trigger chronic inflammatory responses that can exacerbate the underlying injuries and result in reduced performance and impairment of athlete's health ${ }^{(47,56,70)}$. Our results demonstrate that RE triggered an inflammatory response comprising stimulation of production of pro- and anti-inflammatory mediators

A bout of heavy RE triggers a transient inflammatory response stimulating pro- and anti-inflammatory cytokine production ${ }^{(52)}$. Cytokines play an integrative and regulatory role mediating intercellular communication locally or systemically ${ }^{(47)}$. According to our results, the exercise protocol induced inflammation by production of pro-inflammatory molecules IL- $1 \beta$ and TNF- $\alpha$. Exercise induces robust increase of IL- 6 from contracting skeletal muscle, and this myokine stimulates the appearance of antiinflammatory mediators, such as IL-10, besides inhibiting the production of TNF- $\alpha^{(29,52,69,71)}$. It has been reported that factors other than muscle damage, such as stress hormone secretion, increase plasma concentrations of pro-inflammatory cytokines ${ }^{(71,72)}$, which may explain the plasma increase of IL-6 in this study. Moreover, the magnitude and profile of cytokine response to the exercise may differ in relation to changes in systemic $v$. local factors during exercise ${ }^{(64)}$.

IL- 6 has been considered to have a central role in the cytokine response. Enhanced by muscle contraction, IL- 6 release is related to changes in Ca homoeostasis, and mRNA levels of IL-6 in skeletal muscle are increased under conditions of low glycogen ${ }^{(68,71)}$. Nonetheless, the relation between glycogen depletion and cytokine production is not completely clear ${ }^{(73)}$. A limitation in the current study is that muscle glycogen depletion was not analysed, and it could provide new insights. The RE protocol used in the present work promoted high metabolic activity indicated by increased lactate and, therefore, higher IL- 6 concentration in muscle. Higher levels of IL-6 were also observed in plasma of trained and supplemented rats. Pedersen et al. ${ }^{(72)}$ demonstrated that IL-6 induces lipolysis and acts in a hormone-like manner, thus mobilising extracellular substrates or increasing substrate delivery. Although supplemented groups received more energetic substrate, they were associated with lower weight gain, suggesting lipolytic effects of IL-6. However, lower IL-6 levels were observed in EDL muscle of supplemented groups, thus allowing for use of non-lipid fuel supplies.

After eccentric exercise, IL-10 and MCP-1 are increased in an intensity-dependent manner ${ }^{(47)}$ in an attempt to contain the inflammation $^{(58)}$. Produced by a variety of cell types, MCP-1 recruit monocytes into foci of active inflammation and is required for successful muscle regeneration. Impaired muscle regeneration in $\mathrm{MCP}-1^{-/-}$mice suggests an important role for macrophages and MCP-1 in the tissue reparative processes ${ }^{(74)}$. As observed in this study, because of greater metabolic demand and exerciseinduced stress, the concentrations of MCP-1 were increased in the CTRL group. Glutamine administration in both forms was effective to raise plasma concentrations of MCP-1, leading to high capacity of cellular protection. In this sense, increased production of antiinflammatory mediators might limit the production of proinflammatory cytokines associated with prolonged damage ${ }^{(24,29)}$.

Taken together, these findings demonstrate that chronic oral supplementations with L-glutamine and L-alanine in their free form or as a dipeptide induce cyprotective effects mediated by HSP70 responses, following experimental progressive RE and attenuate harmful and inflammatory effects of RE. L-Glutaminecontaining supplements enhanced glutamine availability in plasma and skeletal muscle, which may have stimulated HSP70 higher levels in PBMC and EDL muscle, and reduced DNA-binding activity of NF-kB, supressing inflammation and muscle damage induced by heavy RE.

\section{Acknowledgements}

This work was supported by the São Paulo State Foundation for Research (FAPESP, no. 2012/21087-4 and V. F. C. 2015/00446-4), the Brazilian National Council for Scientific and Technological Development (R. R. sandwich doctorate scholarship, no. 233505/ 2014-8) and the Higher Education and Training Coordination (CAPES)

The present work was designed by R. R., V. F. C. and J. T.; initial manuscript preparation and drafts were prepared by R. R. and revised by V. F. C.; experimental procedures were conducted by R. R., J. S. M. L., T. M. H., A. Y. C.; biochemical and statistical analyses were performed by R. R. and revised by V. F. C.; the final manuscript version was revised by $\mathrm{J}$. T. and P. N.

The authors declare that there are no conflicts of interest.

\section{References}

1. Newsholme P, Procopio J, Lima MM, et al. (2003) Glutamine and glutamate - their central role in cell metabolism and function. Cell Biochem Funct 21, 1-9.

2. Brosnan JT (2001) Amino acids, then and now - a reflection on Sir Hans Krebs' contribution to nitrogen metabolism. IUBMB Life 52, 265-270.

3. Stumvoll M, Perriello G, Meyer C, et al. (1999) Role of glutamine in human carbohydrate metabolism in kidney and other tissues. Kidney Int 55, 778-792.

4. Cruzat VF \& Tirapegui J (2009) Effects of oral supplementation with glutamine and alanyl-glutamine on glutamine, glutamate, and glutathione status in trained rats and subjected to long-duration exercise. Nutrition 25, 428-435.

5. Newsholme P (2001) Why is L-glutamine metabolism important to cells of the immune system in health, postinjury, surgery or infection? J Nutr 131, 2515S-2522S; discussion 2523S-2514S.

6. Singleton KD, Beckey VE \& Wischmeyer PE (2005) Glutamine prevents activation of nf-kappab and stress kinase pathways, attenuates inflammatory cytokine release, and prevents acute respiratory distress syndrome (ards) following sepsis. Shock 24, 583-589.

7. Singleton KD \& Wischmeyer PE (2008) Glutamine induces heat shock protein expression via O-glycosylation and phosphorylation of HSF-1 and Sp1. JPEN J Parenter Enteral Nutr 32, 371-376.

8. Wischmeyer PE (2002) Glutamine and heat shock protein expression. Nutrition 18, 225-228.

9. Senf SM (2013) Skeletal muscle heat shock protein 70: diverse functions and therapeutic potential for wasting disorders. Front Physiol 4, 330

10. Shi Y, Tu Z, Tang D, et al. (2006) The inhibition of LPSinduced production of inflammatory cytokines by HSP70 
involves inactivation of the NF-kappaB pathway but not the MAPK pathways. Shock 26, 277-284.

11. Senf SM, Howard TM, Ahn B, et al. (2013) Loss of the inducible Hsp70 delays the inflammatory response to skeletal muscle injury and severely impairs muscle regeneration. PLOS ONE 8, e62687.

12. Cruzat VF, Rogero MM \& Tirapegui J (2010) Effects of supplementation with free glutamine and the dipeptide alanyl-glutamine on parameters of muscle damage and inflammation in rats submitted to prolonged exercise. Cell Biochem Funct 28, 24-30.

13. Curi TC, De Melo MP, De Azevedo RB, et al. (1997) Glutamine utilization by rat neutrophils: presence of phosphate-dependent glutaminase. Am J Physiol 273, C1124-C1129.

14. Shewchuk LD, Baracos VE \& Field CJ (1997) Dietary L-glutamine does not improve lymphocyte metabolism or function in exercise-trained rats. Med Sci Sports Exerc 29, 474-481.

15. Gleeson M (2008) Dosing and efficacy of glutamine supplementation in human exercise and sport training. J Nutr 138, 2045S-2049S.

16. Rogero MM, Tirapegui J, Pedrosa RG, et al. (2006) Effect of alanyl-glutamine supplementation on plasma and tissue glutamine concentrations in rats submitted to exhaustive exercise. Nutrition 22, 564-571.

17. Lagranha CJ, Hirabara SM, Curi R, et al. (2007) Glutamine supplementation prevents exercise-induced neutrophil apoptosis and reduces p38 MAPK and JNK phosphorylation and p53 and caspase 3 expression. Cell Biochem Funct 25, 563-569.

18. Stoll B \& Burrin DG (2006) Measuring splanchnic amino acid metabolism in vivo using stable isotopic tracers. J Anim Sci 84, Suppl., E60-E72.

19. Zhou X, Wu X, Yin Y, et al. (2012) Preventive oral supplementation with glutamine and arginine has beneficial effects on the intestinal mucosa and inflammatory cytokines in endotoxemic rats. Amino Acids 43, 813-821.

20. Klassen P, Mazariegos M, Solomons NW, et al. (2000) The pharmacokinetic responses of humans to $20 \mathrm{~g}$ of alanylglutamine dipeptide differ with the dosing protocol but not with gastric acidity or in patients with acute Dengue fever. J Nutr 130, 177-182.

21. Rooyackers OE, Soeters PB, Saris WH, et al. (1995) Effect of an enterally administered glutamine-rich protein on the catabolic response to a zymosan challenge in rats. Clin Nutr 14, 105-115.

22. Lima AA, Carvalho GH, Figueiredo AA, et al. (2002) Effects of an alanyl-glutamine-based oral rehydration and nutrition therapy solution on electrolyte and water absorption in a rat model of secretory diarrhea induced by cholera toxin. Nutrition 18, 458-462.

23. Petry ER, Cruzat VF, Heck TG, et al. (2014) Alanyl-glutamine and glutamine plus alanine supplements improve skeletal redox status in trained rats: involvement of heat shock protein pathways. Life Sci 94, 130-136.

24. Cruzat VF, Krause M \& Newsholme P (2014) Amino acid supplementation and impact on immune function in the context of exercise. J Int Soc Sports Nutr 11, 61.

25. Furst P (2001) New developments in glutamine delivery. J Nutr 131, 2562S-2568S.

26. Buyse M, Berlioz F, Guilmeau S, et al. (2001) PepT1-mediated epithelial transport of dipeptides and cephalexin is enhanced by luminal leptin in the small intestine. J Clin Invest 108, 1483-1494.

27. Thamotharan M, Bawani SZ, Zhou X, et al. (1998) Mechanism of dipeptide stimulation of its own transport in a human intestinal cell line. Proc Assoc Am Physicians 110, 361-368.

28. Petry ER, Cruzat VF, Heck TG, et al. (2015) L-Glutamine supplementations enhance liver glutamine-glutathione axis and heat shock factor-1 expression in endurance-exercise trained rats. Int J Sport Nutr Exerc Metab 25, 188-197.

29. Cruzat VF, Bittencourt A, Scomazzon SP, et al. (2014) Oral free and dipeptide forms of glutamine supplementation attenuate oxidative stress and inflammation induced by endotoxemia. Nutrition 30, 602-611.

30. Cruzat VF, Pantaleao LC, Donato J Jr, et al. (2014) Oral supplementations with free and dipeptide forms of L-glutamine in endotoxemic mice: effects on muscle glutamine-glutathione axis and heat shock proteins. J Nutr Biochem 25, 345-352.

31. Harris RC, Hoffman JR, Allsopp A, et al. (2012) L-Glutamine absorption is enhanced after ingestion of L-alanylglutamine compared with the free amino acid or wheat protein. Nutr Res 32, 272-277

32. Goutianos G, Tzioura A, Kyparos A, et al. (2015) The rat adequately reflects human responses to exercise in blood biochemical profile: a comparative study. Physiol Rep $\mathbf{3}$, e12293.

33. Scheffer DL, Silva LA, Tromm CB, et al. (2012) Impact of different resistance training protocols on muscular oxidative stress parameters. Appl Physiol Nutr Metab 37, 1239-1246.

34. Hornberger TA \& Farrar RP (2004) Physiological hypertrophy of the FHL muscle following 8 weeks of progressive resistance exercise in the rat. Can J Appl Physiol 29, 16-31.

35. Prada PO, Hirabara SM, de Souza CT, et al. (2007) L-Glutamine supplementation induces insulin resistance in adipose tissue and improves insulin signalling in liver and muscle of rats with diet-induced obesity. Diabetologia 50, 1949-1959.

36. Bøyum A, Løvhaug D, Tresland L, et al. (1991) Separation of leucocytes: improved cell purity by fine adjustments of gradient medium density and osmolality. Scand J Immunol 34, 697-712.

37. Lund P (1970) A radiochemical assay for glutamine synthetase, and activity of the enzyme in rat tissues. Biochem $J \mathbf{1 1 8}$, 35-39.

38. Nogiec CD \& Kasif S (2013) To supplement or not to supplement: a metabolic network framework for human nutritional supplements. PLOS ONE 8, e68751.

39. Candow DG, Chilibeck PD, Burke DG, et al. (2001) Effect of glutamine supplementation combined with resistance training in young adults. Eur J Appl Physiol 86, 142-149.

40. Singleton KD \& Wischmeyer PE (2007) Glutamine's protection against sepsis and lung injury is dependent on heat shock protein 70 expression. Am J Physiol Regul Integr Comp Physiol 292, R1839-R1845.

41. Santos RV, Caperuto EC \& Costa Rosa LF (2007) Effects of acute exhaustive physical exercise upon glutamine metabolism of lymphocytes from trained rats. Life Sci $\mathbf{8 0}, 573-578$.

42. Cunningham GA, McClenaghan NH, Flatt PR, et al. (2005) L-Alanine induces changes in metabolic and signal transduction gene expression in a clonal rat pancreatic beta-cell line and protects from pro-inflammatory cytokine-induced apoptosis. Clin Sci (Lond) 109, 447-455.

43. Battezzati A, Haisch M, Brillon DJ, et al. (1999) Splanchnic utilization of enteral alanine in humans. Metabolism $\mathbf{4 8}$, 915-921.

44. Hood DA \& Terjung RL (1994) Endurance training alters alanine and glutamine release from muscle during contractions. FEBS Lett 340, 287-290.

45. Wischmeyer PE, Kahana M, Wolfson R, et al. (2001) Glutamine induces heat shock protein and protects against endotoxin shock in the rat. J Appl Physiol (1985) 90, 2403-2410. 
46. Milne KJ \& Noble EG (2008) Response of the myocardium to exercise: sex-specific regulation of hsp70. Med Sci Sports Exerc 40, 655-663.

47. Peake JM, Suzuki K, Hordern M, et al. (2005) Plasma cytokine changes in relation to exercise intensity and muscle damage. Eur J Appl Physiol 95, 514-521.

48. Takayama S, Reed JC \& Homma S (2003) Heat-shock proteins as regulators of apoptosis. Oncogene 22, 9041-9047.

49. Krause M, Heck TG, Bittencourt A, et al. (2015) The chaperone balance hypothesis: the importance of the extracellular to intracellular HSP70 ratio to inflammation-driven type 2 diabetes, the effect of exercise, and the implications for clinical management. Mediators Inflamm 2015, 249205.

50. Barclay CJ, Constable JK \& Gibbs CL (1993) Energetics of fastand slow-twitch muscles of the mouse. J Physiol 472, 61-80.

51. Risøy BA, Raastad T, Hallén J, et al. (2003) Delayed leukocytosis after hard strength and endurance exercise: aspects of regulatory mechanisms. BMC Physiol 3, 14.

52. Ihalainen J, Walker S, Paulsen G, et al. (2014) Acute leukocyte, cytokine and adipocytokine responses to maximal and hypertrophic resistance exercise bouts. Eur J Appl Physiol 114, 2607-2616

53. Wischmeyer PE, Riehm J, Singleton KD, et al. (2003) Glutamine attenuates tumor necrosis factor-alpha release and enhances heat shock protein 72 in human peripheral blood mononuclear cells. Nutrition 19, 1-6.

54. Pahl HL (1999) Activators and target genes of Rel/NF-kappaB transcription factors. Oncogene 18, 6853-6866.

55. Cooper DM, Radom-Aizik S, Schwindt C, et al. (2007) Dangerous exercise: lessons learned from dysregulated inflammatory responses to physical activity. J Appl Physiol (1985) 103, 700-709.

56. Finsterer J (2012) Biomarkers of peripheral muscle fatigue during exercise. BMC Musculoskelet Disord 13, 218.

57. Chen G, Shi J, Qi M, et al. (2008) Glutamine decreases intestinal nuclear factor kappa $\mathrm{B}$ activity and proinflammatory cytokine expression after traumatic brain injury in rats. Inflamm Res 57, 57-64.

58. Vella L, Caldow MK, Larsen AE, et al. (2012) Resistance exercise increases NF- $\mathrm{\kappa B}$ activity in human skeletal muscle. Am J Physiol Regul Integr Comp Physiol 302, R667-R673.

59. Ji LL, Gomez-Cabrera MC, Steinhafel N, et al. (2004) Acute exercise activates nuclear factor (NF)-kappaB signaling pathway in rat skeletal muscle. FASEB J 18, 1499-1506.

60. Urso ML (2013) Anti-inflammatory interventions and skeletal muscle injury: benefit or detriment? J Appl Physiol (1985) 115, $920-928$.
61. Lesueur C, Bôle-Feysot C, Bekri S, et al. (2012) Glutamine induces nuclear degradation of the NF- $\mathrm{KB}$ p 65 subunit in Caco-2/TC7 cells. Biochimie 94, 806-815.

62. Baltusnikas J, Venckunas T, Kilikevicius A, et al. (2015) Efflux of creatine kinase from isolated soleus muscle depends on age, sex and type of exercise in mice. J Sports Sci Med 14, 379-385.

63. Brentano MA \& Martins Kruel LF (2011) A review on strength exercise-induced muscle damage: applications, adaptation mechanisms and limitations. J Sports Med Phys Fitness 51, $1-10$.

64. Hirose L, Nosaka K, Newton M, et al. (2004) Changes in inflammatory mediators following eccentric exercise of the elbow flexors. Exerc Immunol Rev 10, 75-90.

65. Brancaccio P, Maffulli N, Buonauro R, et al. (2008) Serum enzyme monitoring in sports medicine. Clin Sports Med 27, 1-18 vii.

66. Brancaccio P, Limongelli FM \& Maffulli N (2006) Monitoring of serum enzymes in sport. Br J Sports Med 40, 96-97.

67. Wang W, Choi RH, Solares GJ, et al. (2015) L-Alanylglutamine inhibits signaling proteins that activate protein degradation, but does not affect proteins that activate protein synthesis after an acute resistance exercise. Amino Acids 47, 1389-1398.

68. Pedersen BK \& Febbraio MA (2008) Muscle as an endocrine organ: focus on muscle-derived interleukin-6. Physiol Rev 88, 1379-1406.

69. Petersen AM \& Pedersen BK (2005) The anti-inflammatory effect of exercise. J Appl Physiol (1985) 98, 1154-1162.

70. Peake JM, Della Gatta P, Suzuki K, et al. (2015) Cytokine expression and secretion by skeletal muscle cells: regulatory mechanisms and exercise effects. Exerc Immunol Rev 21, $8-25$.

71. Steensberg A, Fischer CP, Keller C, et al. (2003) IL-6 enhances plasma IL-1ra, IL-10, and cortisol in humans. Am J Physiol Endocrinol Metab 285, E433-E437.

72. Pedersen BK, Steensberg A, Keller P, et al. (2003) Musclederived interleukin-6: lipolytic, anti-inflammatory and immune regulatory effects. Pflugers Arch 446, 9-16.

73. Nieman DC, Zwetsloot KA, Meaney MP, et al. (2015) Post-exercise skeletal muscle glycogen related to plasma cytokines and muscle IL- 6 protein content, but not muscle cytokine mRNA expression. Front Nutr 2, 27.

74. Shireman PK, Contreras-Shannon V, Ochoa O, et al. (2007) MCP-1 deficiency causes altered inflammation with impaired skeletal muscle regeneration. J Leukoc Biol 81, 775-785. 